\title{
Pratiques textuelles de l'altérité. Vers une poétique de l'identitaire chez Mona Latif-Ghattas
}

\author{
Cécilia W. FrancIS \\ Université Saint-Thomas
}

Dans la foulée de la critique générée autour de l'écriture migrante, plusieurs essayistes, de Pierre Nepveu à Simon Harel, postulent que la catégorie de textes portant sur l'immigration constitue une topographie symptomatique de la porosité identitaire caractérisant nos sociétés contemporaines. Minoritaire parmi les minoritaires, l'écrivain affecté par la transhumance vit de façon exacerbée le motif de base de la littérature québécoise postmoderne, déterminée par une résistance à l'uniformisation entropique (Gontard, 2007, p. 268). Puisque l'écrivain migrant rappelle l'hétérogénéité 
intrinsèque sous-tendant toute question liée à l'identité culturelle, son texte renvoie à une image à la fois fascinante et inquiétante de la notion d'appartenances (den Toonder, 2008, p. 23), aujourd'hui dépourvue de fondement unitaire. De provenance souvent pluriculturelle, l'écrivain associé à cette condition liminale se trouve "à cheval sur plusieurs cultures, plusieurs pays, plusieurs langues, plusieurs imaginaires » (Robin, 1993, p. 308), et cette diversité originaire le pousse à « des expérimentations formelles pour donner de la cohérence à cette multiplicité et/ou à ces clivages » (ibid.). Dit autrement, une identité composite, plurielle ou hétéroclite s'avère souvent le corollaire d'une «hybridité textuelle» (Dumontet, 2005, p. 84). L'apport premier de cette littérature marquée du sceau de l'exil ou de l'ailleurs se décèle dans sa capacité de déplacer la réflexion sur l'identité culturelle vers la notion d'identitaire, définie par Sherry Simon comme un «lieu problématisé de la rencontre entre culture et identité» (p. 26). Chez nombre d'écrivains migrants, l'identitaire renvoie à «la mise en jeu de l'identité lorsque celle-ci est inassignable, insaisissable, problématique » (Joffrin, 1996, p. 229). Et l'univers fictif s'avère propice à l'exploration de cet identitaire car il autorise les virtualités. Affranchissant l'auteur du pacte autobiographique, mais non de l'interrogation sur l'identité du sujet-écrivain,

La fiction identitaire expérimente les limites de la fictionalisation de la vie de l'auteur en substituant au pacte un contrat de lecture ambigu, qui instaure un rapport de référentialité au cœur d'une fiction. Elle serait une variante de l'écriture autobiographique contemporaine, dans la mesure où elle mêle à la reconstitution rétrospective d'une personnalité l'élaboration par l'écriture d'une identité hétérogène du narrateur après l'expérience douloureuse de l'immigration. (ibid., p. 230) 
Cette littérature se trouve en conséquence façonnée par le déplacement, par «l'exploration de l'espace et la rencontre de l'autre » (den Toonder, 2008, p. 21).

Nous proposons d'examiner ce rapport complexe de référentialité du soi et de l'autre tel qu'il se dessine au cœur de la fiction identitaire de Mona Latif-Ghattas. Nous penchant sur deux œuvres encore relativement peu examinées, à savoir Nicolas, le fils du Nil (1999 [1985]) et Quarante Voiles pour un exil (1986) ${ }^{1}$, nous estimons que l'espace énonciatif de ces deux textes intimistes s'élabore en fonction d'une modélisation esthétique distincte, ancrée dans l'oralité arabo-orientale et ses figurations analogues. Nous désirons montrer dans quelle mesure la quête identitaire esquissée se trouve ainsi prise en charge par des visées de médiation, de coexistence et d'interférences, qui inscrivent les dynamiques intra- et extratextuelles de l'altérité mises de l'avant par l'écriture dans une optique de transculturalité. L'intérêt de notre démarche réside dans l'attention accordée à la facture poétique du texte migrant, à partir d'une perspective analytique sémio-discursive, à l'instar notamment des travaux de Denis Bertrand (2000). Il s'agit d'une approche qui forme la base de notre cadre de travail (Francis, 2003, 2006), qui consiste à interroger les corrélats avant tout intrinsèques (génériques, narratifs, stylistiques, énonciatifs) dans le contexte du récit francophone.

\section{La coprésence des codes orientaux et occidentaux}

Rédigés en début de parcours, Nicolas, le fils du Nil et Quarante Voiles pour un exil s'interpellent dans la mesure où ils sont l'un

\footnotetext{
1 Voir notamment F. Elizabeth Dahab (2009), Lucie Lequin $(1995,1996)$, et Maïr Verthuy et Lucie Lequin (1992).
} 
et l'autre entièrement innervés par l'exploration fragmentaire et elliptique d'éléments identitaires remontant à l'origine, recouvrant l'enfance et la jeunesse liées au pays natal, et témoignent, à partir d'une énonciation empreinte d'inter-culturalité nord-américaine, d'une volonté commune de réappropriation génétique et culturelle. De plus, à la manière des productions littéraires francophones travaillées par des esthétiques métissées (Semujanga, 1999, 2004), ces textes se dérobent aux classements habituels en vertu de leur hybridité formelle - tant générique que langagière. D’après Marc Gontard, le texte francophone « introduit dans le fonctionnement même des formes narratives une hétérogénéité qui remet en cause l'opposition binaire du même et de l'autre, dans un renouvellement postmoderne du discours sur l'altérité » (p. 257).

Eu égard à notre corpus, le premier texte porte comme signalisation générique l'appellation «roman poétique», tandis que le second se déclare "récits et fragments poétiques». Un tel amalgame de pratiques textuelles découle des impératifs d'un discours intimiste fondé sur des assises culturelles orientales et s'écarte pour cette raison, du point de vue du fond et de la forme, des démarches classiques de la prose autobiographique. Précisons, à cet effet, que Nicolas, le fils du Nil fut rédigé en 1979 au Canada et publié d'abord au Caire en 1985, en fonction d'un lectorat égyptien. Les Éditions TROIS republient ce premier roman en 1999 dans sa version originale, augmentée d'une postface de l'auteure. Le second texte, qui a vu le jour en 1986, a paru également chez TROIS, maison d'édition aujourd'hui défunte qui, sous la direction d'Anne-Marie Alonzo, a facilité la circulation d'ouvrages et de travaux expérimentaux, avant-gardistes, assimilés à l'hybridité et à la transculture, qui débordaient les conceptualisations de la littérature québécoise de l'époque. 
Autre trait distinctif essentiel, le geste énonciatif qui soustend ces deux textes est caractérisé par une autoréférentialité renvoyant au dispositif de la voix et au conte arabo-oriental. À la manière d'une charpente posée en immanence, la mise en scène du geste de raconter conditionne dans une large mesure l'œuvre composite de Latif-Ghattas. Qu'on pense aux textes intitulés Les Chants du Karawane (1985), Les Voix du jour et de la nuit (1988), Le Double Conte de l'exil (1990) ou Les Lunes de miel (1996), ce sont surtout des conteurs, des écrivains publics, des figures de poètes ou de cantatrices, des voix d'oracle, des revenants ou des entremetteuses qui peuplent son univers créateur. Chez cette écrivaine originaire d'Égypte, qui immigre au Québec à l'âge de dix-huit ans, il est incontestable qu'un patrimoine originel, nourri des rythmes et des sonorités de l'arabe, langue maternelle, coexistant avec le français, appris dès l'enfance, et d'une culture indissociable des splendeurs du Nil, imprègne son souffle poétique. Transfigurée par l'exil, cette assise culturelle enjoint l'auteure à privilégier l'imbrication dans ses textes de formes génériques et langagières relevant de la rencontre de l'Orient et de l'Occident. "Je suis Orient et Occident. Je n'écrirai jamais que Orient et Occident [...]; c'est une richesse merveilleuse $»^{2}$. En vertu de cette pratique esthétique, Latif-Ghattas rappelle son appartenance à une longue tradition littéraire transmise de façon orale et par les femmes surtout ${ }^{3}$ :

Ma culture arabe est en images, que je le veuille ou non. Le chant circulaire qui me hante est aussi le mien parce qu'il est celui de mon origine et des voix anciennes de ce pays des fresques pharaoniques. La voix des conteurs arabes du désert a

\footnotetext{
2 Latif-Ghattas, dans Grégoire, 1994, p. 32.

3 Kober, 2006, p. 94-95.
} 
sûrement confectionné l'esthétique de mes textes. (LatifGhattas $^{4}$, dans Royer, 1999, p. 74).

Sur cette question, l'auteure a précisé que l'oralité qui circule dans ses textes émane du creuset de ses ascendances multiples, renvoyant certes à la tradition arabo-islamique, mais également aux rituels familiaux et communautaires de la tradition byzantine-orthodoxe et au classicisme gréco-romain, étant donnée sa scolarisation dans le cadre de l'école française (relatée dans son roman Les filles de Sophie Barat), où elle fut lectrice et interprète notamment des tragédies grecques. Un palimpseste à la fois verbal et sensoriel composé d'interférences culturelles enchevêtrées crée ainsi un syncrétisme inusité qui enrichit sa démarche créatrice en langue française.

Observons que son œuvre littéraire s'investit non seulement de l'oralité, mais d'oraliture, concept avancé dans le contexte des études littéraires caraïbes par Maximilien Laroche (1991) pour désigner le substrat des nombreux genres oraux qui participent à la mise en récit d'une culture indigène dominée : mythe, conte, épopée, proverbe, devinette, chanson'. Situé à la croisée de l'oral et de l'écrit, le narrateur de textes francophones s'avère marqueur de paroles, puisqu'il s'ouvre à de multiples destinataires, la coprésence de codes orientaux et occidentaux créant à même le tissu textuel une tessiture vocale métissée, polyphonique. Adoptant une approche anthropologique à l'étude de l'autobiographie dans des

\footnotetext{
${ }^{4}$ Dorénavant, ce nom sera abrégé sous la forme L-G dans les références.

5 Ces données ont été transmises dans une correspondance échangée avec Mona Latif-Ghattas, février 2011.

${ }^{6}$ Latif-Ghattas, dans Grégoire, 1994, p. 33.

7 Beniamino, 2005, p. 142.
} 
contextes interculturels, Hans-Jürgen Lüsebrink signale que l'émergence de l'autobiographie dans les pays africains et arabes montre que les déplacements de frontières entre le privé et le public, entre le non-dit et le dicible, ont généré des formes hybrides, à cheval sur le modèle occidental et les récits de vie inscrits dans des traditions orales. Ces récits de vie tendent à intégrer des formes de dialogisme modelées sur des pratiques de la communication orale quotidienne au cours desquelles le narrateur interagit directement avec son interlocuteur. Les récits autobiographiques des cultures francophones enregistrent donc des données pragmatiques (verbales, nonverbales, contextuelles) inséparables de la scène narrative, ce qui les distingue de l'autobiographie classique, qualifiée par un discours monologique, la distanciation et la linéarité8.

Forte d'un imaginaire infléchi par les rythmes et les sonorités de la langue (dialectale/littérale) et de la culture arabe, Latif-Ghattas partage de nombreuses sensibilités avec d'autres créateurs québécois associés à la transculture, à savoir Naïm Kattan, Nadine Ltaif et Abla Farhoud, qui inscrivent eux aussi dans des écrits hybrides, de manière subversive, dans la substance sonore et gestuelle du sens, des traces d'altérité identitaire. Enrichie par l'adoption d'un nouveau lieu d'énonciation, pétri de pertes et de gains, la filiation orientale incarne chez Latif-Ghattas «les croisements multiples que suscite l'expérience de l'exil » (Nepveu, 1988, p. 234). Cette voix pénétrée d'échos et de couleurs orientaux participe, selon Simon Harel, au décentrement du discours identitaire québécois (p. 86).

${ }^{8}$ Lüsebrink, 2001, p. 104-106. 
Sur ce plan, précisons que la démarche de Latif-Ghattas représente une contribution significative au contre-discours orientaliste qui se développe en Amérique du nord et en Europe. Rappelons que le concept d'orientalisme que l'on doit à Edward Saïd (2005), renvoie au regard réducteur, stéréotypé, exotique, posé par l'Occident sur les cultures africaines, arabes et orientales. Cette lentille ethnocentrique, héritage colonisateur, européen, volontaire ou inconsciente, a eu pour effet de fausser la représentation des cultures arabo-orientales, de réduire la mise en représentation de leurs traditions et pratiques culturelles à un nombre restreint de clichés axés notamment sur la violence, le mysticisme ou l'érotisme. Si en France des auteurs comme Assia Djebar et Leïla Sebbar ont dénoncé la chosification fantasmée du personnage féminin, au Québec, Mona Latif-Ghattas, joignant sa voix à d'autres créatrices de la diaspora (Andrée Dahan, Anne-Marie Alonzo, Nadia Ghalem), a elle aussi critiqué les constructions étroites et binaires de l'orientalisme 9 . En vertu de son geste de raconter à partir de la position culturelle hybride qui est la sienne, elle opère la transmutation du sujet féminin : d'objet observé et décrit, celui-ci se transforme en instance agissante, productrice de discours identitaires singuliers.

\section{Nicolas, le fils du Nil}

Comment la mise en récit du premier texte se déploie-t-elle en termes d'altérité? Nous nous attarderons sur deux composantes de cette modalité discursive en ciblant tout d'abord des

9 Przychodzen, 2006, p. 9-14. 
éléments de genre et de narration et en deuxième lieu, de forme langagière. À cet égard, Nicolas, le fils $d u$ Nil est essentiel dans le parcours de l'écrivaine, puisqu'il en constitue le démarrage et, à la manière d'une palabre du Machrek, annonce l'œuvre à venir. Or pourquoi l'instance narrative choisit-elle d'inaugurer le projet créateur en circonscrivant la médiation du soi par l'Autre, par le père? Plusieurs hypothèses sont valables. D'abord se dire à travers l'autre présuppose une posture de réciprocité, une intersubjectivité attribuable à une fluidité identitaire, traits que plusieurs critiques retiennent en tant que donnée énonciative prédominante de l'écriture autobiographique au féminin ${ }^{10}$. La démarche énonciative du récit de vie est délibérément axée sur une forme d'enclavement, de dédoublement, afin de rendre hommage à une figure influente dans la formation intellectuelle et artistique de la scriptrice. Inscrire une continuité dans une filiation généalogique et physique (Nicolas étant fils du Nil, Mona sa fille est aussi celle $\mathrm{du}$ Nil) permet de transcrire une multi-appartenance, une réciprocité tant familiale que culturelle, géographique ou spirituelle. Qui plus est, adopter la première personne du singulier en tant que femme issue d'un pays arabo-musulman de tradition idéologique patriarcale aurait représenté, pour ce premier texte, un geste de provocation; d'où la nécessité d'édulcorer la prise de position énonciative par la pudeur et la discrétion ${ }^{11}$. Permettant simultanément de s'exhiber et de s'esquiver, cette écriture de soi énoncée par le prisme de l'autre, que nous désignons délibérément auto-biographique pour en souligner l'inhérente polyvalence, relève des pratiques autobiographiques du postcolonialisme, lesquelles

10 Cosslett, Lury et Summerfield, 2000, p. 1-8; Smith et Watson, 1998, p. 9-15.

11 Chaulet-Achour, 1996, p. 291-293. 
échappent aux normes du genre occidental par leur adoption de formes discursives qui amplifient l'entre-deux et le transindividuel ${ }^{12}$. Chez Latif-Ghattas, le positionnement du soi féminin tout comme celui d'autres personnages féminins (grands-mères, épouses, sœurs, filles, gouvernantes) s'observe dans les interstices, s'inscrit en parallèle, sous les branches de l'arbre généalogique (L-G, 1999, p. 39). On décèle sa présence textuelle à l'aide de traces et de fragments : l'autoportrait émerge des «visages de femmes» (p. 49) sculptés par Nicolas, se confond avec l'évocation d'une kyrielle de noms féminins (p. 28), de «bijoux de naissance» (p. 37) offerts en hommage aux nouvelles mères, et renvoie enfin aux eaux féminines du fleuve éponyme par lesquelles s'ouvre et se referme le récit. La fusion identitaire de soi à l'autre paternel émane surtout d'un fonds affectif, d'une interdépendance soudée par l'expérience de l'exil, de la cassure et du deuil communément vécue sur plusieurs plans.

\section{Se dire par l'intermédiaire de l'Autre}

Au niveau de l'anecdote, le morcèlement et la fragmentation de la trame narrative privilégient le parcours du père, mettant de l'avant les diverses particularités qui régissent son statut d'intrus. Le personnage paternel, quoique né au Caire, est le fils d'un père syrien, commerçant venu s'établir dans la vallée des pharaons (L-G, 1999, p. 23). Suivant une coutume familiale, il porte le prénom français de son aïeul et l'accentuation de cette ascendance exogène devient de plus en plus problématique, à mesure que les tensions sociales se font sentir en Égypte au cours des années cinquante et soixante (p.87). On finit par

12 Hornung et Ruhe, 1998, p. 1-3. 
l'associer à la figure de l'étranger, «Pleuvent les mépris Nicolas les voit-il? / "C'est un vendu" disent les siens / "Un étranger" disent les autres siens. / Nicolas ne veut voir il croit. / Il croit » (p. 85). L'équivoque entourant les origines du père s'amplifie, car à l'opposé d'autres entrepreneurs comme lui, et même de son frère Fouad, qui fuient le pays à l'apogée des grands tumultes politiques suscités par Nasser (p. 93-96), il voue une fidélité sans faille à l'Égypte et met en pratique les réformes nationalistes et socialisantes du colonel, bien avant la promulgation des nouvelles lois. Comme pour conjurer son étiquette de paria, il endosse la cause des «fils du Nil» (p. 76), fait preuve d'une indulgence et d'une générosité exemplaires face à ses adversaires, cet altruisme se traduisant en termes arabes par «maalech » (p. 86) :

Nicolas n'est pas voyant il Croit. / «Maalech» pense-t-il. / Maalech. / Ce mot magique « Maalech ». / Qui incite au pardon et à l'indulgence. Mot des nobles de cœur mot des grands des / croyants / des sages. / Il veut dire « laisse aller car l'erreur est humaine », / et la révolution est pure je le sais et j'espère, / car plus fort que l'angoisse plus fort que le mépris / plus fort que l'injustice et plus ancien que l'homme, le Nil. (p. 86)

Se fiant davantage aux valeurs communautaires qu'au cartésianisme et à l'individualisme, le père clame sa légitimité culturelle, incarne par ses gestes et ses attitudes la grande passion sociale égyptienne, celle de la générosité qui précède les contingences du politique et de l'économique (Chebel, 2001, p. 204). Or, sur un autre plan, il perd ses élections en raison de son statut d'entre-deux, malgré une identité légalement attestée (naissance et passeport), «Entre la terre qu'il aime et le nom de ses aïeux, / son Cœur » (L-G, 1999, p. 80), et pour être lui-même un industriel prospère, associé aux «tarbouches» (p. 75), appuyés par le roi Farouk, on le soupçonne de trahison: il 
devient la cible de graves injustices. On confisque son usine de fabrication de soie, on le réduit au niveau d'un "employé syndiqué » (p.107), ce qui lui permet, avec le temps, de se transformer en patron modeste d'une petite société où tous sont travailleurs salariés et syndiqués (p. 107, 125). Victime des transformations sociopolitiques et historiques de son pays, le père accepte son sort et s'engage dignement dans une nouvelle phase de la vie. Mais comme si la déchéance exacerbée par les réformes de Nasser n'était pas suffisante, on assiste à une véritable intensification de la rupture. Celle-ci est attribuée à la fin tragique du père, fauché par un train, un dimanche matin (p. 147-148). Comble des divers drames identitaires tant du côté familial que social, cet incident sous-tend une trame narrative inter-référentielle par laquelle se resserrent les liens entre père et fille.

La mort soudaine du père, précisément au moment où il parvient à surmonter de nombreuses épreuves, et l'intense deuil dans lequel se trouve plongée Mona Latif-Ghattas, ellemême nouvellement immigrée au Québec à l'époque, s'imposent en tant qu'événements catalyseurs, auto-biographiques puisqu'ils transforment à jamais son identité. Cette disparition et la blessure qui s'ensuit déclenchent chez l'auteure la sève poétique. On touche ainsi à la dimension éthique du texte: faire surgir une donnée essentielle du soi à travers l'adversité de l'autre. De la perte et du deuil, décuplés du jour $\mathrm{au}$ lendemain par le décès inattendu d'un père admiré et délaissé en raison de son propre exil, l'écriture éclot, donnant Nicolas, le fils du Nil, mais de façon plus déterminante encore l'ensemble d'un projet créateur ${ }^{13}$. De la catastrophe qui

13 L-G, dans Giguère, 2001, p. 230. 
fait basculer son existence, l'auteure dira qu'elle lui a fait revivre les souvenirs de son propre exil, du départ et du rejet social qu'elle-même a connu au cours des années soixante, à savoir « une douleur [qui] n'a pas de prix » ${ }^{14}$. Le récit de vie médiateur opère la superposition et le télescopage des deuils, jouant ainsi sur divers registres d'intensité pathémique (Bertrand, 2000, p. 265). La mort assimilée à l'exil ravive le traumatisme - tantôt refoulé, tantôt défoulé, mais dont on ne guérit jamais - suscite une intensification de l'imaginaire: " [l]'exil est un choc qui provoque un état de vertige qui à son tour stimule l'imaginaire. Ce choc génère un important travail sur la mémoire »15. C'est dire que « [c]haque nouvelle blessure réanime une ancienne et libère du texte » (L-G, dans Matateyou, 1996, p. 73) : «La première entaille à mon passé fut la mort accidentelle de mon père. [...] Avec lui [Nicolas, le fils du Nil] s'est écrit [sic] en toile de fond l'Égypte, comme si l'un ne pouvait sans l'autre entrer dans le lieu de l'écrit » (ibid.)

\section{Formes langagières : entre oralité et altérité}

Dans Nicolas, le fils du Nil, les péritextes du récit jouent un rôle fondamental dans l'autoréférentialisation de la langue: le premier constitue un métatexte éclairant les motivations qui ont poussé l'auteure à rédiger l'ouvrage (L-G, 1999, p. 9); le second évoque l'importance du registre du réel en Orient (p.11). Attribuables aujeautodiégétique, les deux discours

\footnotetext{
14 L-G, dans Matateyou, 1996, p. 72. L'auteure explique que les événements politiques de l'Égypte des années 1960 l'ont subitement classée (elle et sa famille) parmi les capitalistes qui faisaient fortune au détriment du peuple. 15 L-G, dans Giguère, 2001, p. 222.
} 
liminaires ont pour fonction de fondre la partie biographique, rédigée principalement à la troisième personne, à même le récit intimiste de la mémoire. Il est question de circonscrire le langage et la création en tant qu'éléments de guérison érigés à partir de confluences culturelles, un imaginaire imprégné du sable et de la neige (Lequin, 1996, p. 213). S'inscrit ainsi autrement le signalement rituel du conte, ayant pour fonction de préparer l'auditoire à la venue du récit. $\mathrm{Si}$, dans le premier métatexte, la conteuse revient sur le caractère immuable, impérissable, de la terre d'Égypte à laquelle se greffent les êtres chers évoqués après leur mort, c'est pour assimiler ce devoir mémoriel au geste vital de la parole, à savoir, l'« Histoire de parler d'une Terre, d'un Homme et d'un Nom. / Histoire de parler d'une Mère, d'une Femme et le Nil» (p. 9; nous soulignons). Elle vise ainsi à fusionner les vies et les désignations onomastiques, à les «entremêler au rythme du pays, comme / se mêlent dans la vie la joie et la tristesse » (p.9), et à dissiper ainsi, par anticipation, tout doute susceptible de planer sur l'appartenance culturelle du père qui, grâce à sa conjointe et à sa fille, s'associe au Nil anthropomorphisé ${ }^{16}$. Le sujet d'énoncé réitère de cette manière, à l'aide de l'universalité du conte, la conjonction de sa famille avec un patrimoine identitaire mythique. D’entrée de jeu, il laisse sous-entendre que le récit du père, tout comme le sien, ayant le Nil comme arrière-plan désignatif, sont à jamais soudés par le truchement d'éléments trans-individuels géographiques, historiques, familiaux, affectifs, pragmatiques. Appuyées par une parole qui donne accès à un merveilleux

\footnotetext{
16 Malek Chebel note que le Nil est un «fleuve-roi [...] considéré longtemps comme un dieu, très-saint, père et conservateur du pays, [...] le plus beau, le plus fort » (2002, p. 203-204).
} 
assimilable au «réel» (L-G, 1999, p. 11), tant la voix qui le relate est véridique, ces passerelles de consonance permettent d'atténuer le déliement, la rupture. Et cette volonté de fusion, d'incorporation de l'Autre, se réitère à la toute fin du récit poétique, où, suivant la filiation paternelle, la conteuse finit par emporter son héritage métissé dans ses bagages, afin de l'offrir aux nouveaux visages rencontrés dans le pays nordique : «Moi je leur donne le Nil. Car je l'ai emporté dans mes bagages vers le pays des érables et des neiges j'ai déposé le Nil dans ce pays. / [...] Il paraît que le froid cicatrise les souvenirs » (p. 179).

Effet salvateur, raconter permet de transcender la douleur, et c'est l'importance de l'oralité, du conte et du geste de raconter, rituels conservés de la jeunesse de l'auteure, dominée par la figure de Schéhérazade ${ }^{17}$, qui resurgit dans un appel au lecteur d'adhérer au récit référentiel auto-biographique: «Réalité ou fiction, qu'importe./ Aujourd'hui je raconte pour vous une / histoire d'orient. / En Orient rien n'est fictif. / En Orient même le rêve est réel » (p.11). La mise en exergue intimiste place le récit du père dans un contrat de lecture où la croyance est sollicitée, moins au niveau des enjeux événementiels qu'au plan d'un profil caractériel, du portrait moral et affectif d'un homme, d'un fils, d'un père, voire d'un peuple auquel on s'associe à l'aide d'un autre élément matériel, sonore, et porteur du tiers identitaire : « cet opéra aigu » (p. 17)

\footnotetext{
17 Mona Latif-Ghattas précise : «J'ai été initiée [...] aux codes littéraires de l'Orient par Dada Om Ibrahim, ma gouvernante arabe qui, pendant les mille et une nuits de mon enfance me transmit les contes populaires arabes, leur morale, leur sagesse et leur poésie. Même si, plus tard, j'ai lu les grands poètes arabes, ce sont Ali Baba, Shéhérazade et les autres contes de Dada Om Ibrahim qui laissèrent en moi le plus de traces » (Latif-Ghattas, dans Matateyou, 1996, p. 72).
} 
qui, chanté du haut d'un minaret, monte de la vallée du Nil1s, à la fois mélopée exhalant un deuil trans-personnel et baume à transmettre aux destinataires. D'où l'importance des incipit et excipit encadrant le même énoncé solitaire sur la page blanche en caractères gras, soit « Il était une fois le Nil » (L-G, 1999 , p. 15, 175). Si en vertu d'un même phonème cet énoncé concrétise l'affiliation entre le Nil et Nicolas, il renvoie par ailleurs au il était une fois des contes de fée auquel l'adhésion doit être totale. Un tel encadrement péritextuel installe le récit de Nicolas à l'intérieur d'une continuité circulaire de signifiants dits et chantés, créant ainsi un espace intime de réciprocité et d'inclusion de l'Autre.

Dans son étude consacrée au concept d'interlecte, où il examine le principe de co-énonciation culturelle, de l'interaction entre deux langues, qui régit souvent le contrat de lecture d'écrits francophones, Marc Gontard précise que le roman en tant que genre n'existe pas dans la tradition islamique (p. 257). Celle-ci ne connaît que trois genres narratifs principaux, à savoir le conte (la hikâyât), la biographie (la sîra) et le récit de pèlerinage (la rhila qui se combine avec la maqama, la séance) (ibid.). Dans la culture orale égyptienne, le conte reste une forme narrative des plus riches puisqu'il est indissociable du Coran, dont la genèse implique une transmission orale et répétée qui fut suivie de sa transcription par écrit. D’après Malek Chebel, « la richesse sémantique [du Coran], son esthétisme musical et sonore sont des témoignages quasi suffisants de sa révélation [directe] " (Chebel, 2001, p. 114). Le Livre sacré s'inscrit dans une tradition de la récitation dont le but est de sauvegarder l'authenticité du message.

18 Dahab, 2009, p. 32. 
Dans Nicolas, le fils du Nil, un procédé important du conte oral revient à la présence explicite des narrataires du cadre énonciatif ${ }^{19}$ qui présuppose un cercle élargi d'auditeurs (nommé la halqa). Situés en arrière-plan tout au long du récit, deux vieillards, clients réguliers d'un café maure, discutent et jouent aux dés. Incarnant le passé et le présent ${ }^{20}$, ils sont les témoins observateurs du quotidien, notamment de la vie de Nicolas, en vertu desquels le passage du temps se démarque, surtout par l'entremise de la figure du train : "Passe le train passe le train passe / le train passe / Et passe le petit temps » (L-G, 1999, p. 136). Si on encode en langue turque le «TricTrac » (p. 17) de leur jeu trans-temporel, qui renvoie à la multiethnicité du pays d'origine, les deux vieillards sont assimilés aux archétypes d'une conscience collective, aux sages dotés d'une mémoire historique relativement aux changements sociaux et aux crises ${ }^{21}$. Ainsi renvoient-ils à l'imprévisibilité du destin, au « destin » (p. 141) absurde qui fauche la vie du père bien aimé. Qui plus est, ces deux personnages anonymes, récepteurs tant de «l'opéra aigu » que du conte biographique, préviennent par leur gestuelle de l'imminence de l'accident mortel ${ }^{22}$ et reflètent, par leur situation d'observateurs en retrait,

\footnotetext{
19 Jean Dérive signale qu'il s'agit d'un procédé employé pour donner l'illusion de l'oralité dans le texte, employé par de nombreux écrivains francophones dans leurs œuvres (p. 139-140).

${ }^{20}$ Ces personnages se renouvellent, se remplacent par d'autres, d'où l'idée de temps circulaire et de cycle inter-générationnel dépourvu de transformations linéaires (Latif-Ghattas, 1999, p 41-43).

21 Le positionnement des narrataires suggère la présence d'un «surdestinataire» par lequel on infère un contexte élargi de réception du texte (Charaudeau et Maingueneau, 2002, p. 560-561).

22 «Au café c'est au tour de Celui qui tient les dés. / Va les lancer mais attend attend attend / car il entend entend entend / monter monter monter / aigu aigu aigu / monter de la vallée du Nil / L'Autre regarde Celui qui n'a plus l'air
} 
la position perceptive dédoublée de la conteuse ${ }^{23}$, oscillant entre énonciatrice et sujet d'énoncé, à qui revient la narration du conte oriental.

Renvoyant à l'oralité à la fois implicite et explicite du texte, une forte sollicitation de la dimension sonore du langage et des figures assimilables à la musique dans Nicolas, le fils $d u$ Nil explique en grande partie la simplicité apparente du texte et l'épuration de la forme, trait reconnu de la poésie arabe ${ }^{24}$. Édouard Glissant a souligné, à cet effet, que les textes francophones marqués par l'oralité négligent souvent l'essentiel du réalisme occidental, esquivant la description des paysages, la leçon des décors, la motivation des personnages, pour mieux mettre en valeur la symbolique des situations. C'est comme si ces textes s'efforçaient de déguiser sous le symbole, de dire en ne disant pas; le critique et auteur dénomme cette tendance à la métonymie une pratique du détour ${ }^{25}$. Cette technique narrative qui conjugue une retenue au plan de l'action narrative avec un déferlement de signifiants sonores permet à la narratrice d'imposer un effet de flux identitaire, de porosité de soi : elle se représente de manière effacée, comme si justement cet autoportrait moins langagier que sonore formait le palimpseste du tableau inclusif de soi-même et de l'Autre.

de voir / que l'Autre attend que Celui / figé / lance les dés » (Latif-Ghattas, 1999, p. 147).

23 Dans une note éditoriale pour l'édition de 1999 aux Éditions TROIS, AnneMarie Alonzo précise que le récit fut publié une première fois en 1985 à compte d'auteur au Caire dans le but d'aider à financer la construction du Foyer Evergreen à Hélouan, maison de retraite pour les aînés dont les enfants avaient émigré à travers le monde et qui se retrouvaient seuls en Égypte. Les deux narrataires vieillards sont ainsi assimilés aux aînés subissant les conséquences de l'exil de leurs enfants et de leurs petits-enfants.

24 Ltaif, 2001, p. 25.

25 Glissant, 1990, p. 82-83. 
On observe, en effet, au plan de la structure narrative de l'intrigue, une sorte de dépouillement événementiel du vécu du père, une condensation chronologique au plan des actions marquée par les temps forts de l'existence : naissance, études, débuts dans l'entreprise, fiançailles, mariage, naissance des enfants. Le resserrement anecdotique caractérisé par l'absence de pauses explicatives ou de synthèses trahit la facture éminemment pragmatique d'un texte qui minimise l'importance du décor physique et renvoie aux qualités oniriques et mythiques du conte universel. Assimilé à une poétique de l'hybride, ce genre de pratique discursive fait ressortir un chronotope de l'intemporel et de l'universel égyptien. De plus, il a pour résultat d'amplifier la densité sensorielle et sensible du texte, laquelle met à vif les souvenirs de la narratrice. Reprendre ainsi la vie de Nicolas permet de restituer à l'aide d'une mémoire corporelle tout un univers cyclique partagé qui rythme le quotidien millénaire au Caire. À titre d'exemple, synecdoque de fruits accompagnés de leurs parfums et couleurs, les saisons sont dépeintes au temps présent, leur arrivée scandée par le chant du fruitier ambulant: oranges en hiver (L-G, 1999, p. 44), abricots au printemps (ibid.), mangues, raisins, pastèques en été (p. 45) et dattes à l'automne (ibid.). À l'instar du conte arabe, on assiste à un déploiement de la métaphore et de l'allégorie par lesquelles s'exprime un discours intimiste voluptueux ${ }^{26}$. Un tel symbolisme dans le déroulement cyclique ${ }^{27}$ d'un univers visuel, olfactif et gustatif, s'imprimant

\footnotetext{
26 Ltaif, 2001, p. 24.

27 Mona Latif-Ghattas voit une extension de cette notion de cycle à même la prosodie de sa démarche littéraire: "Je dirais que la rythmique orientale est circulaire alors que la rythmique occidentale est plus linéaire. La langue française est plus linéaire alors que la langue arabe est celle des contes. C'est le mélange des deux qui fait mes textes » (Latif-Ghattas, dans Giguère, 2001, p. 228).
} 
au plan sensible de l'être, constitue une sorte d'arrière-fond cognitif intarissable de rituels et d'habitudes de vie, retransmis d'une génération à l'autre (L-G, 1999, p. 44-46). D’où la nécessité de réactualiser cette mémoire corporelle, car « [c]et appel aux sens redistribue le poids de l'exil, de la rupture, du métissage ou du pays entre l'intellect, l'affect et le sens » (Lequin, 1995, p. 136).

$\mathrm{Au}$ plan de la phrase et sa syntaxe, on rencontre régulièrement les glissements de la troisième à la première personne, le passage du discours rapporté au discours direct et vice versa, afin de mimer un brouillage identitaire, une multiplicité vocale.

Nicolas écoute toujours et n'écoute jamais car plus / fort est le Nil, je me dois à ses bords et puis c'est / mon devoir allons-y bien sûr il se lance. Il se lance / et la valse commence et le fou tourbillon / enveloppe ses mots ses désirs ses actions, Nicolas / veut rassembler tous ceux qui lui ressemblent / pense-t-il, moi pour vous tous pour le Nil. (L-G, 1999, p. 78)

Un autre trait caractéristique de l'oralité reste la morphologie nominale employée, soit la manière de désigner les personnages relevant du giron familial : baba (pour père), mama, dada (pour gouvernante) sont toujours présentés sans pronoms personnels. Jamais la narratrice n'énonce un syntagme comme : «le père de Nicolas », «la mère de Nicolas », « sa gouvernante ». Si cet effet rappelle la simplicité rhétorique du conte, il a surtout pour fonction de brouiller l'ordre générationnel impliquant les relations et les appartenances, renforçant ainsi une réciprocité immédiate entre père et fille. Dans son étude portant sur les textes maghrébins francophones, Nadine Le Duff remarque que la suppression de l'article défini essentialise, «va de pair avec l'accumulation 
parataxique, qu'on peut interpréter comme un refus de la démarche de rationalisation. Le récit annote le réel, saisit des instantanées, et gagne une rapidité d'exécution qui dramatise la scène » (p. 57).

Sur le plan de la facture énonciative, on peut apprécier l'originalité de cette poétique du conte lorsqu'on la compare avec un autre texte intimiste de Latif-Ghattas, plus contemporain, soit son récit d'enfance écrit en dialogue avec Louise Desjardins(2004), où l'on revient sur la donnée familiale en détail, mais où la rhétorique du conte cède à une voix expressément autobiographique accompagnée d'un usage conventionnel des démarcations pronominales et temporelles.

On remarque que la forte dimension musicale du récit détient une semblable fonction de rapprochement identitaire. Phénomène suprasegmental essentiel du signifiant textuel (lorsque le sens est saisi en tant que flux modulatoire avant la catégorisation), la musicalité en tant que figure, métaphore et description, joue un rôle compensatoire dans la syntaxe narrative relâchée, traversée de non-dits et de blancs. La richesse sonore se dégageant du texte provient d'une pléthore de sources : chants et mélodies présentés en sourdine, dictons reproduits, proverbes, expressions répétées, slogans politiques, lexèmes arabes ou turcs, onomatopées, hymnes religieux, bruits ambiants reconstitués, lettres et voyelles répétées, étirées. Un tel continuum auditif actualise les valeurs rythmiques, voire les tonalités musicales, dont s'imprègne la vie individuelle et collective, cette trame sonore étant à la fois modulée de vibrations, de souffles et de silences, attributs traduisant une forte présence déictique, si typique du conte arabe. Commentant cette transposition d'une structure-culture arabe 
en langue française, la poète Nadine Ltaif déclare que les auteurs de provenance arabe «n'écriv[ent] pas si ce n'est une musique de mots» (p.25). La musicalité crée une épaisseur sensible qui rappelle l'univers chromatique de l'enfance. Préférant la circularité à la linéarité, elle réduit la distance entre énoncé et énonciation, relègue au second plan la nécessité d'une mémoire événementielle étoffée. Cette altérité matérielle, qui nécessite la récitation du texte, permet de rejoindre le père, le passé, le Nil, moins par un effort du logos que par une forme de mutualisme affectif et corporel. Il s'agit d'une dimension précognitive du sens, grâce à laquelle se superposent non seulement les méandres du Nil, mais aussi et surtout les intonations de l'« opéra aigu » qui, tel un chœur abolissant la distance entre auditeurs passés, présents et futurs, annonce les crises et soulage les blessures. C'est ainsi que l'identitaire chez Mona Latif-Ghattas s'affirme en tant que "processus relationnel, le moi se constituant dans les moments dialogiques avec l'altérité, soit celle d'un-e autre choisi-e, soit celle d'un espace social particulier» (Lequin, 1995, p. 134).

Renvoyant à la tradition arabe de la prosodie ${ }^{28}$, la trame sonore participe à une configuration autoréfléchissante enclavée par laquelle l'écriture auto-bio-graphiquepropose d'autres niveaux de recoupement entre père et fille. Rappelons qu'en plus de ses réussites au plan technologique et commercial, Nicolas possède des dons artistiques. Il peint,

\footnotetext{
${ }^{28}$ La création poético-musicale populaire au Proche et Moyen-Orient, qui date de la période pré-islamique de ces régions, serait liée à la figure du poètemusicien, qui joue un rôle de divertisseur public, d'artiste créateur, de conservateur des traditions de son milieu et de commentateur des événements courants. Il est appelé sa'er - poète —, qawwal - celui qui dit, ou faiseur de paroles -, hadi-faiseur de hida'(genre poético-musical) (Shiloah, 1974, p. 52-63).
} 
dessine, sculpte, chante et joue de l'oued oriental (L-G, 1999, p. 49). Qui plus est, il est l'auteur d'un livre politique ( $C E T T E$ TERRE EST $\grave{A}$ NOUS», p. 77) et d'un recueil de poèmes ( $L A$ RÉVOLUTION DE L'ÂME», p. 85), titres inscrits en majuscules dans le texte, comme si cette activité intellectuelle et créatrice, posée en amont du deuil, soudait à jamais les affinités entre père et fille, contenait en germe la destinée artistique de la fille. Les talents du père, être cultivé et sensible, sont reçus par la fille comme un héritage précieux, ce qui situe ce texte à l'encontre de la tendance des récits de la seconde génération d'immigrés dont la transmission s'avère souvent aléatoire, cousue de tensions et de silences (Viart, 2009). En tant que scriptrice-conteuse, le sujet d'énoncé émerge et prend corps dans le texte, non seulement en vertu de sa densité sensorielle, mais surtout à l'aide d'une posture énonciative qui lui permet de se fondre dans une altérité ouverte et plurielle, mouvement qui s'inscrit dans un processus de recherche et de reconstruction identitaire continu. On retient de cette relation filiale l'influence déterminante de la figure paternelle, qui facilite chez la fille une entrée dans le monde des signes et du chant, dans l'univers symbolique de la langue (Saadi, 2007, p. 12). À l'intérieur d'une culture où les femmes sont subordonnées au genre masculin, la générosité remarquable de Nicolas, à la fois à l'égard de sa fille et de sa conjointe, appuyant leur épanouissement personnel, demeure l'un des traits dominants de ce conte hommage auto-bio-graphique ${ }^{29}$.

\footnotetext{
29 Associées aux discours autobiographiques empreints de l'exil postcolonial, Assia Djebar (2007) et Leïla Sebbar (2003) ont avoué une dette envers leur père en ce qui concerne leur évolution en tant qu'intellectuelle et écrivaine. De manière générale, les écrivaines ressortissantes de cultures islamiques sont nombreuses à exprimer leur redevance à la figure du père cultivé (Sebbar, 2007).
} 


\section{Quarante Voiles pour un exil}

L'héritage paternel transculturel et trans-temporel sous-tend également le second récit de vie de Mona Latif-Ghattas, fût-ce dans une inscription plus abstraite. Le thème du questionnement identitaire s'intensifie, s'élabore en fonction d'une problématique permettant de faire converger deuil personnel et deuil collectif. Dans Quarante Voiles pour un exil, on approfondit l'interdépendance entre mort et création, entre mort et vie à l'aide d'une structure qui mime un ruban de Möbius, symbole de circularité à l'infini. Le récit, composé de prose et de fragments poétiques, se divise en quatre parties distinctes, chacune portant un sous-titre ayant trait, par glissement de sens, au lexème éponyme « voile ». S'opérant par gradients $d u$ voile à la voile, la métamorphose générique se fait sur l'élan d'une série de biographèmes dédoublés, enchevêtrés. Une narratrice qui dit je revient sur son enfance égyptienne laquelle est mise en parallèle avec un épisode tragique de l'histoire de la Nubie ancienne. Le retour créateur sur cette région disparue de la Haute Égypte chevauche simultanément les débuts de l'exil à Montréal.

Or qu'en est-il de la fonction esthétique de l'oralité dans cette démarche? La stratification de l'intrigue, où se télescopent jeunesse et maturité, est rendue d'autant plus prégnante qu'elle s'érige à partir d'un dialogisme intratextuel permettant de circonscrire des altérités culturelles modélisant l'identitaire chez la narratrice. Ce dialogisme s'établit d'abord avec une troisième voix, celle de Nour, figure androgyne et flottante, embrayeur de l'acte créateur : « Je dis Nour parce que Nour est prénom qui dans ma langue natale peut être signé par un lui ou par un elle. [...] Si Nour est un lui c'est un lui 
rempli d'elle. Transformé par une elle en son lui véritablement Humain » (L-G, 1986, p. 48-49).

Cette relation imaginaire nouée avec le passé égyptien, incarné par Nour, permet de capter une autre facette de l'oralité dans le texte latifghattasien. Le transfert et la réciprocité ainsi suggérés entre le je et le tu, le masculin et le féminin, l'ici et l'ailleurs de l'acte énonciatif a pour effet d'ouvrir le texte à une polyphonie inclusive d'un(e) Autre, oublié(e) ou méconnu(e). Par les sortilèges de son verbe, la narratrice parvient ainsi à libérer la parole étouffée de la Nubie engloutie sous le lac Nasser, celle d'un peuple égyptien exproprié, pour exhaler le deuil de cette perte d'héritage millénaire auquel se mêlent les douleurs de sa propre distanciation par rapport au pays d'origine.

Deuxièmement, l'inscription de l'oralité adopte une forme résolument intertextuelle. À la fois incipit et substrat signifiant embrassant l'ensemble du récit, une palabre de l'Orient, retranscrite, souligne la nécessité de l'épreuve du deuil, tout en invitant au retour à la vie au bout de quarante jours :

En Orient le deuil dure quarante jours

Et quarante nuits.

Les hommes laissent pousser leur barbe.

Les femmes se voilent.

Le jour nous entoure d'amis et de sollicitude pour nous aider

à traverser le temps présent du deuil.

La nuit dans sa solitude ramène tous les deuils ancestraux. [...]

Les quarante nuits de deuil sont des nuits blanches.

Le quarantième jour une voile perce au loin.

On écarte le voile.

Si on a l'aptitude à la vie ${ }^{30}$. (p. 39)

30 Il s'agit d'une variante de l'incipit: «Voile-toi quarante jours ma fille pour consommer le deuil / de ce qui est perdu / Pleure sous tes voiles et lamente- 
Faisant écho au titre, ce dicton arabe qui s'érige en un deuil chanté, renvoie aux quarante jours de deuil, d'isolement, de jeûne et d'arrachement qui suivent la disparition de l'être cher. Un tel rituel s'impose dans Nicolas, le fils $d u$ Nil à la mort du père (p. 154) et réapparaît dans Quarante Voiles pour un exil à l'occasion du deuil familial, vécu à la suite de la perte d'un enfant. Le second texte identitaire parle donc du soi et de ses doubles familiaux, ancestraux, d'un deuil multiple et composite, dont la conjuration se manifeste au moment d'intégrer un nouvel espace de vie. L'arrière-plan sensoriel des paysages désertiques inspire ainsi un travail créateur se produisant sur des «page[s] de neige» (L-G, 1986, p. 97), permettant au sujet énonciateur de se donner un ancrage au pays d'accueil. Si la palabre en tant que véhicule du souffle poétique prend en charge le récit auto-bio-graphique de la formation artistique chez la scriptrice, c'est le deuil collectif indissociable de la parole (libérée) de Nour qui commande le mouvement vers l'altérité extratextuelle.

\section{Les voiles de l'enfance. La fracture du quotidien}

Dans la première partie de Quarante Voiles pour un exil, soustitré à deux endroits respectivement «Assouan l'année de mes six ans » et «Assouan l'hiver de mes six ans » (p. 13, 22), la trame narrative est prise en charge par une fracture esthétique $d u$ quotidien ${ }^{31}$, grâce à laquelle la fillette accède à l'altérité au

toi bien / Griffe-toi la face et panse les éraflures / Mais le quarantième jour lève tes voiles / La voile attend » (p.9). D'autres versions et reprises de la palabre scandent le texte du début jusqu'à la fin.

31 Greimas, 1987. 
niveau des sens. L'épisode s'actualise sous le signe de la transmutation inhérente à « la voile », car il s'agit de l'inversion de l'ordre événementiel de la palabre liminaire. À partir de la perspective du sujet d'énoncé autobiographique, on relate une aventure singulière, remplie de félicité, laquelle se superpose au deuil parental, motif premier du séjour familial au Cataract Hotel à Assouan, lieu paradisiaque de villégiature hivernale pour les classes aisées et aristocratiques du monde entier. Dans ce contexte, on constate un nombre limité d'événements transformateurs, dont le plus important est la concrétisation de la relation entre le sujet d'énoncé et Ali Lawi, simple serviteur nubien, qui agit en substitut paternel.

La formation artistique de la jeune protagoniste se fait sous l'égide d'un pseudo père, un "colosse noir aux dents d'ivoire, vêtu d'une robe blanche de coton amidonnée » (L-G, 1986, p. 15), parlant «une langue inconnue familière et indéchiffrable. / Une langue en ébène » (p.19) qui l'initiera au monde des merveilles faisant partie du patrimoine égyptien enseveli sous les sables désertiques de la Nubie. Sans frontières véritablement tracées, ce territoire a appartenu à un peuple ancien dominant le Nil bien « avant les pharaons » (ibid.). La singularité des expériences que vit la fillette revient au fait que son apprentissage se fait dans cette région vulnérable, à la veille de disparaître sous les eaux, suite à la construction du barrage d'Assouan de 1957 à 1970 ${ }^{32}$. Durant les quarante jours que dure le deuil de sa mère, Ali Lawi emmène la fillette en excursions (secrètes) d'Assouan à Edfou et encore plus loin, « vers le point des merveilles » (L-G, 1986, p. 19). Lors de ces excursions, elle s'engage dans une expérience sensorielle unique, un champ de

32 Fogel, 1995. 
présence sensible ${ }^{33}$, où le corps, la conscience et la perception sont continuellement éveillés par la découverte de phénomènes matériels d'un monde hétérogène, méconnu et sur le point d'être englouti. La narratrice met l'accent sur une sorte de motricité perceptive, analogue à l'inchoativité du processus créateur. Plusieurs sens chez la fillette sont sollicités dans ces fractures qui se détachent de la prévisibilité du quotidien commandée par les «feloukes sur le Nil» (L-G, 1986, p. 20). Que ce soit par la perception visuelle «[d]es cannes à sucre, [...][d] es maisons de briques beiges et des palmiers » (p. 16) (la vue), l'écoute du «rire de Ali Lawi sur le Nil bleu d'Assouan » (p. 15) (l'ouïe), le rapprochement physique, « [m]a main perdue dans la main de Ali Lawi nous marchons dans la poussière à l'heure où les adultes ainsi que mes parents font la sieste » (ibid.) (le toucher), ou le mouvement corporel, « [j]e trotte près de lui dans la chaleur il marche lentement mais ses pieds sont si grands » (ibid.) (la kinesthésie), il s'agit d'expériences précognitives enregistrées au niveau d'une mémoire corporelle, qui se veulent autant d'incitations préverbales à l'acte créateur.

Comme si ce parcours initiatique permettant d'apprivoiser la beauté sauvage et millénaire de la Nubie à la veille de son anéantissement ne constituait pas en soi un don précieux, l'hiver des six ans du sujet d'énoncé en referme bien plus. Ali Lawi emmène Mona dans un lieu secret «trop délabré pour les touristes » (p. 22), où se trouve, sous « deux marches de granite recouvertes de sable rouge » (ibid.), le passage vers une maison souterraine. Cette habitation millénaire, nommée la "maison d'éternité », dans laquelle on pénètre en soulevant une dalle de granite est associée aux mystères du Nil, « [p]arce que le fleuve

33 Merleau-Ponty, 1990, p. 30. 
de l'éternité coule sous sa terre» (p. 32). Éblouie, la fillette y découvre des fresques nubiennes «inestimables» (p.20), les quatre murs de la maison étant couverts de dessins d'une immense valeur archéologique - des poules, des fleurs, des scorpions colorés et l'autoportrait d'un enfant (dont le sexe reste indéterminé). Ali Lawi déterre une foule d'artefacts nubiens parmi lesquels on compte des poupées, des scorpions, des coffres de bois et une urne, qu'il offre à la fillette, lui disant que «tous [c]es objets [...] contiennent des messages » (p. 27), « renferment des contes » (p. 26). De tous ces artefacts insolites évoquant les contes classiques des Mille et une nuits, " la lampe d'Aladin, la potiche des quarante voleurs, le bateau de Sinbad, le trésor du roi Karoune » (p. 29), il en est un qui s'en distingue. Il s'agit d'« une petite statuette [déterrée] qui pleure. / Du coin de sa tête fêlée un bout de tissu froissé émerge. [...] / Il ne faut pas tirer le vieux bout de tissu de la tempe fêlée » (p. 26). Présage de la déterritorialisation collective à venir et de l'exil du sujet d'énoncé, cette statuette obscure et lourde de sens au plan symbolique est liée, elle aussi, au processus créateur en germe. Une fois rentrée à l'hôtel, Mona raconte des histoiresà la statuette: «[j]e lui raconterai l'histoire d'une fillette couverte de bijoux qui pleure de joie le jour de ses noces » (ibid.). Ainsi concrétise-t-elle les conjectures d'Ali Lawi, à savoir qu'« un jour [elle lira] plus couramment que le mufti du quartier. / [Qu'elle écrira] sur de longs parchemins interminables» (p. 27). Attendu que cette mise en marche du geste de raconter représente l'aboutissement de tout un parcours perceptif déterminant, il n'est pas étonnant qu'elle s'opère dans la proximité des voiles, dès lors tout intérieures et abstraites, cette métamorphose étant représentée par le bas du rideau en orgonza dans la chambre « gonflant comme une voile» (p. 26). 
Indissociable d'une paternité culturelle génératrice des mystères de la parole ${ }^{34}$, ce fragment de l'enfance porte des traces sensorielles appartenant à une civilisation millénaire, peu avant son engloutissement. Dans cette expérience esthétique, la réalité extérieure prend sens et valeur sur un mode quasi fusionnel. C'est comme si le contact avec le parfum des objets culturels découverts pouvait rendre le sujet pleinement présent au monde et le monde intelligible immédiatement. Comme le note Landowski, « [l]a convocation du sujet par la forme immanente des figures du monde sensible coïncide en ce cas avec la révélation du sens » (1997, p. 234). Sur le plan de la perception du monde naturel, on circonscrit ainsi une sorte de décalage actualisé par l'apparition soudaine de certaines configurations qui attirent le sujet hors de luimême et provoquent un retour à soi : "l'événement esthétique » (Greimas, p. 14) apparaît comme un accident, mais un accident qui éclaire le regard en révélant «quelque chose qui ressemble à un ordre » (ibid.) : la présence du sens. Cette interdépendance subjective de formes hétérogènes dont la rencontre était improbable, fonctionne comme une mutualité momentanée, fait émerger une vérité de l'être, une révélation où le sujet se découvre tout à coup autre. En tant que (rare) témoin de ce monde qui n'est plus, la future scriptrice aura pour tâche d'en perpétuer la mémoire. Au-delà de l'euphorie de l'anecdote, la partie initiale du texte pose en immanence la coexistence des contraires (le/la voile), les expériences extraordinaires relatées étant inséparables d'un deuil à la fois collectif et personnel, à la source d'une parole poétique sur le point de s'éclore.

34 Dans son livre La Triste Beauté du monde (1993), Mona Latif-Ghattas fait une dédicace à Anne Hébert en hommage à sa poésie. 


\section{Le chant du deuil interpersonnel}

C'est avec le décalage du temps et de l'espace que s'affirmera la valeur des artefacts nubiens rescapés de l'oubli. Dans « La voile du destin » (L-G, 1986, p. 33-36), qui présuppose le propre exil de la narratrice, celle-ci relate qu'on lui transporte les artefacts par navire du Caire à Montréal dans des valises ayant appartenu à Nicolas, le père. En vertu du mystère et de l'histoire tragique qu'ils renferment, ces objets aussi rares qu'hétéroclites - « [s]tatuettes, fétiches, scorpions, scarabées, potiches et coffrets» (p. 40), s'ajoutant aux « [p]etits bouts de papyrus émincés chargés de signes » (p.48) - suscitent par leur matérialité même une inclination poétique. Le sujet d'énoncé se donne pour mission d'incarner le vécu auquel ils se rattachent à l'aide de paroles de Nour qu'elle vise à restituer : « [e]ntailler, décrypter et transcrire l'œuvre de Nour» (p. 98). Or pourquoi cette insistance sur le devoir de mémoire qui au fond ne pourrait être qu'une interprétation approximative? D'une part, revenir sur des données méconnues et obscures relevant de son patrimoine identitaire l'aide à se comprendre à mesure qu'elle confronte son propre exil: «Comment interpréter ces idéogrammes désarmants. [...] Quand je me penche sur leur reflet ils me renvoient mon vieux visage » (p. 61). D'autre part, s'attardant sur la figure de Nour, prénom qui en arabe suggère la lumière et qu'elle donne à la statuette à la tête fêlée (p. 103), la narratrice désire immortaliser un chapitre qu'elle estime déplorable de l'histoire égyptienne, et faire partager aux lecteurs du pays adoptif, la nostalgie d'un pays perdu. Portée par une réciprocité engageant Nour, grâce à laquelle la narratrice vise à rejoindre l'Autre, à savoir les narrataires de sa parole, cette démarche biofictive s'avère 
extrêmement complexe et ardue. C'est à Régine Robin que nous empruntons le terme de biofiction pour désigner cette composante essentielle de l'acte énonciateur de Quarante Voiles pour un exil. Dans L'Immense Fatigue des pierres (1996), l'écrivaine se sert de ce concept en tant que (ré)invention littéraire du passé, laquelle caractérise plusieurs écrits de la survivance, à la fois idéalisée et douloureuse, permettant aux survivants des atrocités historiques de confronter une histoire familiale ou culturelle, dont ils sont privés, pour en faire leur deuil. L'auteure vise ainsi à dévoiler, à travers les récits de vie où la réalité s'interpénètre de fiction, la part d'imagination à l'œuvre dans toute réappropriation du passé. Chez LatifGhattas, la transposition biofictive du deuil jointe à ses multiples ramifications ancestrales, ethnographiques et historiques suscite et stimule un travail poétique qui permet de conjurer la détresse de l'état divisé.

Or l'intérêt premier revient à circonscrire les destinataires de la parole de Nour: «De qui parle Nour exactement. A qui et en quel temps. Je cherche désespérément les dédicaces » (L-G, 1986, p. 74). La narratrice vise ainsi à approfondir ses connaissances sur l'histoire des membres de la communauté nubienne qui, à la suite de leur expropriation, ont été non seulement disséminés sur des terres éloignées du Nil, mais relocalisés aussi au Soudan, pays voisin ${ }^{35}$. Pour faire avancer ses recherches, la narratrice adulte effectue un voyage de retour à Assouan, sur la trace ethnographique d'Ali Lawi, mais lorsqu'elle le retrouve enfin, habitant un territoire éloigné de $s a$ « mer » (L-G, 1986, p. 81), le $\mathrm{Nil}^{{ }_{3} 6}$, celui-ci ne parle plus, ne

\footnotetext{
35 Fogel, 1995.

36 Malek Chebel rappelle que le nom arabe du Nil (Bahr an-Nîl) renferme effectivement le mot « mer » (2002, p. 203).
} 
rit plus, son état d'aphasie ayant été sans doute provoqué par le tumulte d'un exil forcé. Et quoique suivant d'abord une méthode scientifique - se servant d'une loupe, d'une lampe et de plusieurs dictionnaires dans son exercice de décryptage - la narratrice finit par choisir d'«entailler» (p. 40) ces artefacts « inusables » et « fragiles » (ibid.) « qui sentent la boue du Nil » (p. 49), d'autant plus précieux qu'ils furent rescapés de justesse de l'inondation. La procédure de travail adoptée évoque en ce sens la notion de blessure: "[t]outes les blessures se ressemblent [...], [ont] le souvenir de l'entaille» (p. 55). L'entreprise poétique se situe non pas dans le cadre d'une rationalité objective, mais adopte plutôt une logique empirique, viscérale, associée aux données intuitives. Une fois les blessures ravivées, celles-ci débouchent sur diverses strates d'expériences oblitérées, n'ayant jamais atteint le stade du dicible. «J'entaille./ Des objets friables./ Statuettes et potiches / Je fais une légère incision. / J'extrais le message. [...] / J'interprète les signes. / Et les signes parlent toutes les langues » (p.54). Palper, tâter, sentir et ressentir, voilà ce qui permet de faire « suinte[r] » (p. 49) le deuil du soi et de l'autre : «Qui ne risque pas le mal du souvenir / N'aura jamais vécu » (p. 51).

S'appuyant sur la sagesse orientale de la palabre liminaire, la narratrice se rend compte dans son travail de poète à Montréal qu'il lui faut, en effet, vivre une période d'ascèse, soit «quarante temps d'arrachement»(p.61), quarante jours de recréation biofictive afin de restituer l'œuvre de Nour. Du point de vue du positionnement spatial, on observe que le décryptage poétique obéit à une trajectoire bachelardienne, suivant une verticalité allant du bas vers le haut. La narratrice passe en effet de sa table de travail, un meuble en érable construit au Québec, 
située au sous-sol de sa demeure, au deuxième étage, où les fenêtres de son bureau laissent pénétrer la luminosité du ciel montréalais. Cette trajectoire, inspirée de sa réalité d'immigrée, reflète les couches de complexité en jeu, puisque la narratrice cherche à extraire et à décoder autant de bribes du préconscient que de réalités touchant à la mémoire et aux souvenirs conscients. Répartie sur quarante nuits blanches, l'œuvre à restituer reprend ainsi l'histoire du peuple nubien.

[L]e peuple de Nubie est si ancien qu'il ne mourra jamais. / Qu'il n'est pas de ceux qui se laissent engloutir sous des lacs artificiels. / Qu'au bout de quarante jours de peine il prit la voile qui passait sur le Nil vers la nouvelle terre. / Qu'il replantera. / Car depuis la nuit des temps il porte ses souvenirs. (p. 82)

Dans l'errance et l'exil, les «souvenirs » constituent en effet l'unique chez soi, la véritable maison habitée, portée au niveau de l'âme, à savoir l'oikos impérissable, susceptible de «permettre [...] l'établissement d'une "résidence" endopsychique» (Harel, 2005, p.132). Il s'agit d'une composante identitaire s'appliquant non seulement à la narratrice mais à tous ceux qui sont marqués par la transhumance. Évoquer les souvenirs d'un peuple ancestral lié à son propre lignage possède un effet salutaire, car on parvient ainsi à conjurer ses propres démons, à consolider ses multiples appartenances identitaires.

\section{Du dialogisme intratextuel à l'altérité extratextuelle}

Le discours restitué de Nour forme le cœur de la troisième partie éponyme, «Quarante voiles pour un exil » (L-G, 1986, p. 37-100). On parvient dès lors à capter la parole de la figure 
androgyne qui s'intercale et se démarque par une typographie distincte (caractères gras) à l'intérieur du récit métanarratif. Ces deux trames textuelles interagissent à la manière de vocalises chantées en duo, donnant accès à un dialogisme dramatique ayant son propre rythme et ses propres variations tonales. Or, contrairement à Nicolas, fils $d u$ Nil, il ne s'agit plus d'histoires narrées, mais d'une trentaine de poèmes cryptiques qui traduisent l'indignation et la douleur faisant partie d'un traumatisme trans-générationnel : "Comme on retire la gaze sur une plaie séchée. / J'ai capté la couleur./ La forme quelquefois et surtout la musique. / Dans l'ordre où je les ai reçues d'une halte à l'autre de ma / propre vie » (L-G, 1986, p. 98).

D'où l'importance de la facture purement sonore de ces bribes poétiques et de leur association à la musique et au chant. Les références renvoient au «mawal» (p.45) (une plainte chantée), au requiem (p.47), à l'oratorio (p. 64), à l'opéra (ibid.), aux tam-tams (p.58), aux "gammes de musique» (p. 92), aux notes musicales (p. 93), à « un livre à lire ou à chanter» (p. 103). La dimension sensible de la musique, sa capacité d'évoquer l'émotivité et d'influer sur l'humeur du récepteur, s'approfondit en vertu d'un contrepoint syntagmatique accompagnant chaque poème. Signe de l'abjection d'une collectivité, d'une douleur insondable, l'expression « la nuit de Nuij » (p. 42) subit plus d'une vingtaine de variantes; à titre d'exemples, «Nuij au seuil de la nuit» (p. 45), « Nuij la nuit de l'insomnie » (p. 51), « Nuij dans le deuil de la nuit» (p.65), « Nuij dans le secret de la nuit» (p. 66), etc. Ce jeu subtil de reprise et d'altération au niveau des phonèmes se veut non seulement une mise en écho du vocable "nuit», renvoyant au moment de l'exode des Nubiens de la vallée du 
Nil, mais aussi la trame d'une lamentation qui refuse de s'achever : "Le gros dictionnaire brun à l'écorce écaillée dit que la Nubie / a été engloutie village après village. / Lentement au rythme du cours du Nil. / Il dit aussi que Ouadi Halfa a sombré doucement au mois / d'Avril dans le cœur de la nuit» (p. 81). On le voit, l'essentiel des paroles poétiques de Nour ne réside pas dans leur cohérence, mais dans le tracé sensible qui s'en dégage, soutenu par la dimension purement sonore du texte, allant d'une violence et d'une déchéance qui atteignent leur acmé dans "Oratorio pour un orient blessé » (p.64), à la guérison et à la célébration de la résilience, exprimées dans « Poème pour le vent » (p. 88) et « Poème actif » (p. 89).

$\mathrm{Au}$ bout de ses « quarante descentes dans la pénombre de la lumière » (p. 99), la narratrice trouve enfin, au moment de la «quarantième voile» (p.100) (dans la quatrième partie du texte), les dédicaces de Nour, ce qui lui permet d'associer cette parole rescapée à un geste de donation, à une retransmission à l'intérieur d'une nouvelle collectivité, celle du Québec, pour y inscrire sa propre altérité. Un tel travail est rendu possible parce que la narratrice finit par céder «son cœur à l'état oriental » (p. 104), à donner libre cours aux impulsions premières de son propre idiolecte empreint d'oralité. Du coup, elle renoue avec une "humeur de sagesse qui accueille la fatalité et même l'irréparable, mais aussi [...] surtout l'insondable vibration d'un chant d'éternité » (ibid.), susceptible d'appuyer son effort de régénération. Voilà pourquoi cette restitution biofictive se trouve en dernier sous-tendue par la métaphore de la voile qui s'assimile dès lors aux réalités du pays d'accueil. La quarantième voile, celle qui débouche sur la vie (après le deuil), se concrétise par la figure du vent et des feuilles volantes remplies d'écriture, «incrustées aux quatre 
coins » (p. 100) de la pièce de travail à Montréal (l'une des feuilles s'est même réfugiée sur le cadre entourant le portrait de son père). Comme quoi le vent nordique et énergisant de Montréal, qui «retournait dans [s]on jardin la neige des anciennes tempêtes » (ibid.), contribuait au souffle créateur qui s'affirme dans le pays adoptif.

Pénélope a achevé sa merveilleuse tapisserie, / Shéhrézade ses contes fabuleux, Ali Lawi m'a donné / Nour, et moi je relègue au temps dans le couvert d'un livre à lire ou à chanter. / [...] Et je t'ai embelli. / Et je me suis embellie en retour. / Voilà pourquoi je te chuchote en fin de rêve. / Ce matin j'ai trouvé la clé des chants. (p. 103-104)

Destinées aux lecteurs du pays des neiges, les pages des livres à venir encodées du " chant » oriental, comme des voiles qui circulent de l'enfance à l'âge adulte, finissent par s'avérer un puissant antidote aux affres de l'exil.

\section{Pour conclure}

Dans son ouvrage intitulé Écritures migrantes et identités culturelles, Clément Moisan (2008) réitère et approfondit ses réflexions sur le pluralisme culturel du Québec (amorcées dans Ces Étrangers du dedans) en élaborant une conceptualisation hétérogène de la littérature québécoise contemporaine, cette métamorphose éthique et esthétique étant attribuable non seulement aux influences de la mondialisation, mais surtout à « la présence d'écrivains venus d'ailleurs dont l'apport est singulier, différent et diversificateur à bien des points de vue » (2008, p. 118). Cette vision élargie, post-nationaliste, repose sur le fait que les écrivains migrants 
ont contribué à créer des occasions inédites de médiation identitaire. Leurs œuvres littéraires sont des sources de « restructuration des identités en contact, par divers modes d'appropriation, d'acculturation, de métissage» (p. 72). Or comment cette médiation par la différenciation, par l'altérité, se manifeste-t-elle? D'après Moisan, l'altérité repose sur la présence de deux termes différents, à savoir le moi et l'autre, qui doivent s'interrelier, entrer en relation, dans des échanges symboliques, de l'ordre du discours :

Pour que l'altérité fonctionne, atteigne une complémentarité, il faut que l'image de l'Autre soit traduite de chacun des deux points (de vue), de moi et de l'autre. La comparaison des deux figures de l'Autre fait apparaître des différences qui peuvent aussi devenir l'objet de discours. (ibid., p. 94)

S'opérant par méandres et détours, la saisie de singularités identitaires que l'on doit aux écritures migrantes fait en sorte qu'elles participent directement à la création de transferts culturels et, partant, à la transculturalité ${ }^{37}$, laquelle résulte en une «mise en mouvement radicale de l'identité qui ne laisse plus rien de stable dans le sujet » (Ouellet, 2003, p. 18).

Nous l'avons observé, la démarche de Mona Latiff-Ghattas s'érige en une paratopie traversée par la présence de l'Autre, du double, où l'espace énonciatif travaillé par une coprésence perpétuelle de l'ici et de l'ailleurs s'articule en termes de nostalgie par rapport au pays (pré)pharaonique, tout en étant marqué en contrepoint par les registres de la dysphorie (arrachement, deuil) et de l'euphorie (découverte, conciliation). Ce continuum émotif composite adopte la figuration du conte, du dicton, de la présence et de la musicalité de la voix, ces

37 Ashcroft, Griffiths, Tiffin, 2007, p. 213-215. 
configurations de l'oralité transposées sur le plan du signifié et du signifiant étant simultanément une ouverture vers l'Autre, le destinataire de cette parole métèque. Voilà remodelés selon une poétique propre à cette auteure les dispositifs du mouvement et de l'errance, caractéristiques reconnues des écritures migrantes (Nepveu, 1988, p. 234). Le projet créateur de Mona Latif-Ghattas présuppose de cette manière une confluence d'expériences qui devient une sorte d'entre-deux, voire un entre-plusieurs, où l'échange permet à l'écrivain migrant de prendre ses distances par rapport à sa culture d'origine et à la culture d'accueil «en décontextualisant la première et en contextualisant la seconde", mais aussi, en lui donnant «l'occasion de réaborder de manière positive des valeurs qui s'étaient trouvées désincarnées et de se situer par rapport à la culture de la société d'accueil » (Moisan, 2008, p. 100). Mona Latif-Ghattas déclare à cet effet : " j'ai intégré avec le temps les merveilleux paysages de mon pays d'adoption ainsi que les préoccupations qui sont les siennes et qui sont aussi devenues les miennes » (L-G, dans Metateyou, 1996, p. 74). L'auteure a concrètement matérialisé cette forme de vie médiatrice, rendant sa démarche non seulement interculturelle, mais transculturelle. Un métissage poétique s'opère chez elle notamment à partir du recueil de poèmes, La Triste Beauté $d u$ monde, où " Concerto pour une île » célèbre son hybridité identitaire, où le Nil, par une surimpression de géographies, se mêle aux eaux du Saint-Laurent :

Je te regarde Montréal / Du lever au coucher des saisons / [...] Et quand le Saint-Laurent comme une rame inoxydable / ou un néon bleuté défie les brumes et les neiges / Je te regarde Montréal / [...] Et je me dis que tes saisons circulent enfin / Dans mes désirs. (L-G, 1993, p. 99-100) 
En 1994, l'auteure collabore avec Louise Desjardins et Jean-Paul Daoust à la rédaction d'un recueil singulier. Intitulé Poèmes faxés, celui-ci consiste en un enchaînement circulaire de poèmes commis une année durant, une sorte de tête-à-tête à trois, une " nouvelle trinité ${ }^{38}$, facilitant l'enchevêtrement de sensibilités et d'imaginaires interpersonnels et transculturels, échangés par télécopie à toutes les trois semaines et portant les diverses indications de date et de lieu d'expédition auxquelles on peut s'attendre dans ce genre de correspondance. Un texte subséquent, ayant pour titre Momo et Loulou (2004), rédigé conjointement par Mona Latif-Ghattas et Louise Desjardins, est un récit d'enfance raconté en tandem, structuré en échos et en filiations, permettant aux interlocutrices de vivre l'épreuve de l'autre, bref de (se) «conaît[re] ${ }^{39}$ individuellement et à l'unisson, en vertu d'un dialogisme inusité, créé entre le ciel du Caire et celui de Noranda, malgré les milliers de kilomètres qui séparent les expériences fondatrices. Enfin, dans le poème, «On est de quelque part», l'auteure se déclare de la "grande Cité humaine » (L-G, 2004, p. 107) et évoque un espace créateur qui englobe et transcende décors et appartenances.

De cette jonction nommée exil, je fais de la littérature [...] Avec mes citoyennetés qui me dédoublent et qui me fragilisent

Souvent de part et d'autre incomprise J'écris du lieu de la méconnaissance Et de la reconnaissance

Pour vivre utile Éternellement transfuge de mes cités (L-G, 2004, p. 110).

\footnotetext{
38 Daoust, dans Daoust, Desjardins et Latif-Ghattas, 1994, p. 7.

${ }^{39}$ Merleau-Ponty, 1990, p. 245.
} 
Ces exemples concrets de transferts identitaires, transposés dans la dimension illocutoire qui sous-tend l'ensemble du projet créateur de Latif-Ghattas, offrent de façon corollaire, au lecteur (à l'écrivain) natif du Québec l'opportunité de plonger dans un espace identitaire diversifié, intermédiaire, «où l'identité doit être reconsidérée, recomposée, comme s'il devait désormais en recoller les morceaux fragmentés depuis qu'il a accédé au [...] vécu interculturel » (Moisan, 2008, p. 100). C'est ainsi que l'œuvre littéraire de Mona Latif-Ghattas constitue un terreau exceptionnel d'exploration et d'interrogation des diverses strates de l'altérité, des processus identitaires par lesquels se construisent, se déconstruisent et se reconstruisent les représentations du soi et de l'autre.

\section{Bibliographie}

ASHCROFT, Bill, Gareth GRIFFITHS, Helen TIFFIN (2007 [2000]), Post-Colonial Studies. The Key Concepts, Oxon, Routledge, deuxième édition.

BEnIAMINo, Michel (2005), "Oraliture », dans Michel Beniamino et Lise Gauvin (dir.),Vocabulaire des études francophones. Les concepts de base, Limoges, PULIM, p. 142-145.

Bertrand, Denis (2000), Précis de sémiotique littéraire, Paris, Nathan.

Charaudeau, Patrick et Dominique Maingueneau (2002), Dictionnaire d'analyse du discours, Paris, Seuil.

Chaulet-Achour, Christiane (1996), «Autobiographies d'Algériennes sur l'autre rive : se définir entre mémoire et rupture », dans Martine Mathieu (dir.), Littératures 
autobiographiques de la francophonie, Paris, L'Harmattan, p. 291-308.

Chebel, Malek (2001 [1995]), Dictionnaire des symboles musulmans. Rites, mystique et civilisation, Paris, Albin Michel.

- (2002 [1993]), L'Imaginaire arabo-musulman, Paris, Quadrige/Presses universitaires de France.

Cosslett, Tess, Celia Lury et Penny Summerfield (dir.) (2000), "Introduction », dans Feminism and Autobiography. Texts, Theories, Methods, London/New York, Routledge, p. 1-21.

DAHAB, F. Elizabeth (2009), Voices of Exile in Contemporary Canadian Francophone Literature, Lanham/Boulder/New York/Toronto/Plymouth, UK, Lexington Books.

DAOUST, Jean-Paul, Louise DESJARDins et Mona LATIF-GHATTAS (1994), Poèmes faxés, Trois-Rivières, Écrits des Forges, coll. «Écrits des Forges poésie».

DEN TOONDER, Jeanette (2008), «La mondialisation de l'écriture migrante », dans Marie Carrière et Catherine Khordoc (dir.), Migrance comparée/Comparing Migration. Les littératures du Canada et du Québec/The Literatures of Canada and Québec, Bern/Berlin/Bruxelles/New York, Peter Lang, p. 19-36.

DÉRIVE, Jean (2005), « Oralité », dans Michel Beniamino et Lise Gauvin (dir.), Vocabulaire des études francophones. Les concepts de base, Limoges, PULIM, p. 139-140.

Desjardins,Louise et MonaLATif-GhatTAS (2004),Momo et Loulou, Montréal, Remue-ménage.

DJEBAR, Assia (2007), Nulle part dans la maison de mon père, Paris, Fayard.

DumONTET, Danielle (2005), «Hybridité textuelle/Effets de texte - Hybridité linguistique/effets de langue dans les textes des "écritures migrantes" au Québec», dans Hafid Gafaïti, Patricia M. E. Lorcin et David G. Troyansky 
(dir.), Migrances, diaporas et transculturalités francophones, Paris, L'Harmattan, p. 83-105.

FoGEL, Frédérique (1995), «Des Nubies, des Nubiens : traditions scientifiques et locales de l'identité ", Égypte/Monde arabe, Première série, no 24, [en ligne], mis en ligne le 8 juillet 2008, http://ema.revues.org/index634.html.

FRANCIS, Cécilia W. (2003), "Lecture sémiotique et récit identitaire francophone au féminin ", Revue de l'Université de Moncton, "La théorie littéraire et sa pratique à travers le roman francophone », vol. 34, nos 1 et 2, p. 31-64.

- (2006), Gabrielle Roy, autobiographe. Subjectivité, passions et discours, Québec, Presses de l'Université Laval, coll. «Intercultures ».

GIGUÈRE, Suzanne (2001), Passeurs culturels. Une littérature en mutation, Québec, Presses de l’Université Laval.

Glissant, Édouard (1990), Poétique de la Relation, Paris, Gallimard.

GONTARD, Marc (2007), "Francophonie et globalisation. La question de l'interlecte», International Journal of Francophone Studies, vol. 10, nos 1 et 2, p. 253-269.

GRÉGOIRE, Monique (1994), «Mona Latif-Ghattas: de l'exil à l'appartenance», Nuit blanche, le magazine du livre, no 55 , 1994, p. 30-34.

Greimas, Algirdas J. (1987), De l'imperfection, Périgueux, Éditions Pierre Fanlac.

HAREL, Simon (2005), Les Passages obligés de l'écriture migrante, Montréal, XYZ.

HoRnung, Alfred et Ernstpeter RuHE (dir.) (1998), "Préface», dans Postcolonialisme et autobiographie. Albert Memmi, Assia Djebar, Daniel Maximim, Amsterdam/Atlanta, Rodopi, p. 1-4. 
JOFFRIN, Laurence (1996), « La fiction identitaire dans l'écriture migrante au Québec : Présentation liminaire », dans Martine Mathieu (dir.), Littératures autobiographiques de la francophonie, Paris, CELFA/L'Harmattan, p. 223-231.

KoBer, Marc (2006), «Femmes écrivaines/femmes écrites. Récits au féminin dans l'Égypte au XX siècle », dans Carmen Boustani et Edmond Jouve (dir.), Des femmes et de l'écriture. Le bassin méditerranéen, Paris, Karthala, p. 94-95.

LANDOWSKI, Éric (dir.) (1997), Lire Greimas, Limoges, Presses universitaires de Limoges.

LAROCHE, Maximilien (1991), La Double Scène de la représentation. Oraliture et littérature dans la Caraïbe, Québec, GRELCA/Université Laval.

LATIF-GHatTAS, Mona(1985), Les Chants du Karawane (traversées poétiques), Le Caire, Elias Publishing House.

- (1986), Quarante Voiles pour un exil, Laval, Éditions TROIS.

- (1988), Les Voix du jour et de la nuit, Montréal, Boréal.

- (1990),Le Double Conte de l'exil, Montréal, Boréal.

- (1993), La Triste Beauté du monde, Montréal, Noroît.

- (1996), Les Lunes de miel, Montréal, Leméac.

— (1999 [1985]), Nicolas, le fils du Nil, Laval, Éditions TROIS.

- (1999), Les filles de Sophie Barat, Montréal, Leméac.

- (2004), « On est de quelque part », Possibles, vol. 28, no 1 , hiver 2004, p. 106-110.

LE DuFF, Nadine (2005), « De la créolité au rythme, du rythme au chaos, et retour... un essai de lecture des textes francophones maghrébins ", Expressions maghrébines, vol. 4, no 1, été, p. 35-61. 
LEQUIN, Lucie (1995), "Mona Latif Ghattas: une mélopée orientale dans un espace blanc ", Québec Studies, no 19, p. 133-141.

- (1996), "Quand le monde arabe traverse l'Atlantique », dans Lucie Lequin et Maïr Verthuy (dir.), Multi-culture, multiécriture. La voix migrante au féminin en France et au Canada, Paris, L'Harmattan, p. 209-217.

LTAIF, Nadine (2001), « Attraction-répulsion de l'origine » dans Lucie Lequin et Catherine Mavrikakis (dir.), La francophonie sans frontières. Une nouvelle cartographie de l'imaginaire au féminin, Paris, L'Harmattan, p. 23-27.

LÜSEBRINK, Hans-Jürgen (2001), «Dynamiques de l'autobiographie. De l'ancrage anthropologique aux horizons interculturels", dans Robert Dion, Frances Fortier et Élisabeth Haghebaert (dir.), Enjeux des genres dans les écritures contemporaines, Québec, Nota bene, p. 103-120.

Matateyou, Emmanuel (1996), «Entretien avec Mona Latif Ghattas : Écrivaine de l'exil », Études francophones, vol. 11, no 1 , p. 71-82.

MERLEAU-PonTy, Maurice (1990 [1945]), Phénoménologie de la perception, Paris, Gallimard.

MoISAN, Clément (2008), Écritures migrantes et identités culturelles, Québec, Nota bene.

MoISAN, Clément et Renate HILDEBRAND (2001), Ces Étrangers du dedans. Une histoire de l'écriture migrante au Québec 19371997, Québec, Nota bene.

NEPVEU, Pierre (1988), L'Écologie du réel. Mort et naissance de la littérature québécoise contemporaine, Montréal, Boréal.

OuELLET, Pierre (2003), L'Esprit migrateur. Essai sur le non-sens commun, Montréal, Trait d'union. 
PrzYchodzen, Janusz (2006), "Identités hybrides. Orient et orientalisme au Québec », préface à Mounia Benalil et Janusz Przychodzen (dir.), Identités hybrides. Orient et orientalisme au Québec, Montréal, Paragraphes, p. 9-14.

RoBIN, Régine (1993), «Introduction : un Québec pluriel », dans Claude Duchet et Stéphane Vachon (dir.), La Recherche littéraire. Objets et méthodes, Montréal, XYZ, p. 301-309.

- (1996), L'Immense Fatigue des pierres. Biofictions, Montréal, $\mathrm{XYZ}$.

RoYer, Jean (1999), «Mona Latif Ghattas. De l'exil à l'écriture ", Écrivains contemporains. Nouveaux entretiens, Montréal, Trait d'union, p. 73-75.

SAADI, Nourredine (2007), "Père et fille », dans Leïla Sebbar (dir.), Mon père, Montpellier, Éditions Chèvre-feuille étoilée, p. 7-9.

SAÏD, Edward (2005 [1978]), L'Orientalisme. L'Orient créé par l'Occident, Paris, Seuil.

SEBBAR, Leïla (2003), Je ne parle pas la langue de mon père, Paris, Julliard.

- (dir.) (2007), Mon père, Montpellier, Éditions Chèvre-feuille étoilée.

SEMUJANGA, Josias (1999), Dynamique des genres dans le roman africain: Éléments de poétique transculturelle, Paris, L'Harmattan.

- (2004), « La mémoire transculturelle comme fondement du sujet africain chez Mudimbe et Ngal », Tangence, vol. 75, été, p. 15-39.

SHILOAH, Annon (1974), «Le poète-musicien et la création poético-musicale au Moyen-Orient », Yearbook of International Folk Music Council, vol. 6, p. 52-63. 
SimoN, Sherry (1991), " Espaces incertains de la culture », dans Sherry Simon, Pierre L'Hérault, Robert Schwartzwald et Alexis Nouss (dir.), Fictions de l'identitaire au Québec, Montréal, XYZ, p.13-52.

SMITH, Sidonie et Julia WATSON (dir.) (1998), "Introduction », dans Women, Autobiography, Theory. A Reader, Madison, University of Wisconsin Press, p. 3-52.

VerthuY, Maïr et Lucie LEQUin (1992), «Sous le signe de la pluralité : l'écriture des femmes migrantes au Québec », Tessera, "L'autre regard/Other Looks. Representation. Race et/and Gender », vol. 12, été, p. 51-59.

VIART, Dominique (2009), « Le silence des pères au principe du "récit de filiation"», Études françaises, vol. 45, no 2, p. 95-112.

\section{Résumé}

Consacrée à l'analyse de deux textes de Mona Latif-Ghattas, à savoir Nicolas, le fils du Nil et Quarante Voiles pour un exil, la présente étude part de la notion d'identitaire (Sherry Simon) pour montrer dans quelle mesure cette interrogation littéraire du soi s'élabore en fonction d'une esthétique ancrée dans l'oralité arabo-orientale qui s'accompagne de figurations analogues, par lesquelles la pratique scripturale de l'auteure dévoile des traces de l'altérité. Axé sur la facture poétique du texte migrant, l'article divulgue que la quête identitaire esquissée se trouve ainsi prise en charge par des visées de médiation, de coexistence et d'interférences qui influent sur les dynamiques intra- et extratextuelles de l'altérité mises de l'avant par l'écriture. 


\begin{abstract}
Based on an analysis of Mona Latif-Ghattas' texts entitled Nicolas, le fils du Nil and Quarante Voiles pour un exil, this article sets out to explore the notion of identitaire (Sherry Simon), in order to examine the ways in which this literary investigation of self is permeated by Arabic-Oriental orality, accompanied by analogous aesthetic representations, by which the author's literary works disclose traces of otherness. In scrutinizing the poetic dimension of the migrant text, the article goes on to reveal that the author's identity quest adopts processes of mediation, coexistence and conjunction, which affect the intra- and extra-textual dynamics of otherness as developed in the writing.
\end{abstract}

